\title{
Investigaciones realizadas en la Universidad de Aveiro sobre caracterización mecánica de las construcciones existentes en adobe en Portugal y propuestas de rehabilitación y refuerzo. Resultados alcanzados
}

\begin{abstract}
Outputs from the research developed at the University of Aveiro regarding the mechanical characterization of existing adobe constructions in Portugal and proposals for rehabilitation and strengthening
\end{abstract}

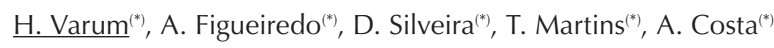

RESUMEN

SUMMARY

En Portugal, sobre todo en la región de Aveiro, las estructuras de fábrica de adobe fueron el sistema constructivo predominante durante la primera mitad del siglo XX. En la actualidad es aún significativo el legado del patrimonio - cerca de 30\% de las construcciones existentes - el cual está formado por un conjunto significativo de construcciones de elevado valor histórico y arquitectónico. En las últimas décadas se ha descuidado su rehabilitación, que ha redundado en el estado actual de daño en muchas de estas construcciones, con desfavorables condiciones de seguridad, y en algunos casos comprometiendo su uso. No obstante, en los últimos años, se observa un creciente interés, asociado a factores ambientales, económicos y de salvaguarda del patrimonio, tanto por parte de las administraciones locales como en el ámbito de los inversores privados, por la conservación y rehabilitación de estas construcciones. Ante esta nueva realidad, que pone de manifiesto la necesidad de desarrollar soluciones y técnicas de intervención que permitan, a través de la rehabilitación y/o refuerzo, prolongar la vida útil de estas estructuras, en la Universidad de Aveiro un grupo de investigadores viene desarrollando un trabajo multidisciplinar sobre las construcciones de adobe en Portugal. El objetivo principal del mismo se centra en la creación de una base de resultados experimentales que apoye las intervenciones de rehabilitación y/o refuerzo del patrimonio edificado, y el desarrollo de soluciones de mejora de su comportamiento estructural. Esta investigación se constituye como un guía que procura contribuir al conocimiento de estas fábricas, enfocado sobre todo a la rehabilitación de las mismas, aportando información que permita a los diferentes profesionales que trabajan en este ámbito actuar en la conservación de este patrimonio.

113.107

Palabras clave: construcciones históricas, adobe, fábrica, caracterización mecánica, refuerzo sísmico.
In Portugal, mainly in Aveiro region, adobe buildings were the prevailing construction system during the first half of the 20th century. Presently, the legacy that results from the patrimony built in that period is still significant-about $30 \%$ of the existing constructions in the region- and, moreover, it is comprised by a large number of patrimonial buildings of high historical, cultural and architectural value. A marked negligence in the rehabilitation of this patrimony has been observed in the last decades, and has resulted in the actual damage state presented by many of these constructions, which generally resulted in unfavourable safety and durability conditions. However, in the last years, an increasing interest in the conservation and rehabilitation of the adobe patrimony in the region, associated with environmental, economical and patrimonial safeguard factors, has been demonstrated by public administrative entities and also by the private sphere. In face of this reality, which emphasizes the need and importance of developing intervention strategies that may enable, by rehabilitation and/or strengthening, extending the lifespan of these structures, a group of researchers of the University of Aveiro has been developing a multidisciplinary study on the adobe construction in Portugal. The main objective of this research work is the creation of a test results database that may support rehabilitation and/or strengthening interventions, and also the study of solutions for the improvement of the structural behaviour of these constructions. In this sense, the research carried out was constituted seeking to contribute to the knowledge of this type of adobe masonry, focused mainly on their rehabilitation, providing information to the professionals and institutions working in the field of heritage conservation.

Keywords: historical constructions, adobe, masonry, mechanical characterization, seismic strengthening.
Informes de la Construcción Vol. 63, 523, 127-142, julio-septiembre 2011 ISSN: 0020-0883 elSSN: $1988-3234$ doi: 10.3989/ic. 10.016

\footnotetext{
(*) Universidade de Aveiro. Aveiro (Portugal)

Persona de contacto/Corresponding author: hvarum@ua.pt (H. Varum)
}

Fecha de recepción: 02-03-10 Fecha de aceptación: 20-09-10 
1-8. Ejemplos de construcciones de adobe en la región de Aveiro
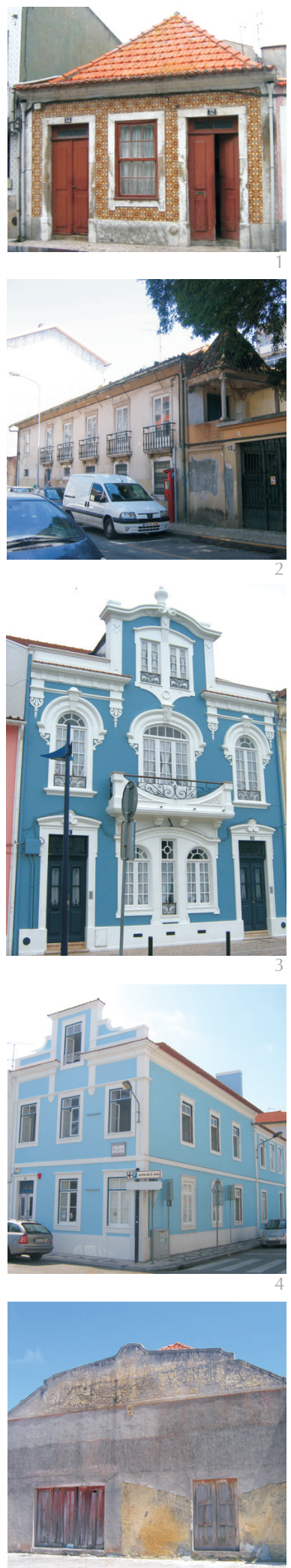

\section{INTRODUCCIÓN}

En Portugal la construcción con tierra, sobre todo en adobe y tapial, se encuentra extendida prácticamente por todo el territorio. No obstante, el empleo de dichas técnicas estuvo desde siempre condicionado por la naturaleza del suelo característico de cada zona. Así, mientras que en el sur del país es el tapial la técnica más difundida, en la franja norte atlántica, con mayor predominancia en la región de Aveiro, el adobe es la técnica más empleada. En esta región, la construcción de estructuras de fábrica de adobe fue el sistema constructivo principal fundamentalmente durante la primera mitad del siglo XX. Así que, en el transcurso de ese período la fábrica de adobe fue utilizada en diversas tipologías constructivas que respondían a las necesidades de la población de entonces, desde construcciones rurales de una sola planta, hasta edificios urbanos de diversos usos (edificios residenciales, edificios militares, almacenes industriales, iglesias, etc.), de gran superficie y de más de una planta, y hasta incluso otros tipos de estructuras como pozos de agua, muros de contención de tierras, etc.

El legado del patrimonio construido durante ese periodo es todavía muy significativo en la actualidad, cerca de un $30 \%$ del total de construcciones existentes en la región son de adobe, formadas además por un elevado número de elementos patrimoniales inmuebles de elevado valor histórico, cultural y arquitectónico, como son de ello ejemplo algunos de los edificios estilo Art-Nouveau. No obstante, desde el abandono del empleo del adobe en las construcciones de la región, se ha observado un notable descuido en la conservación y rehabilitación del patrimonio existente. Como resultado de esta actitud de negligencia generalizada, se constata el estado actual de degradación y deterioro acusado presentado por gran parte de estos edificios. Esta situación condujo a que, ante el estado actual del patrimonio construido, en general, la demolición haya sido la medida adoptada para muchos de estos edificios. No obstante, en los últimos años, debido fundamentalmente a factores de orden eco- nómico y de responsabilidad ambiental y de salvaguardia del patrimonio, se observa un creciente interés, tanto de parte de las administraciones como en el ámbito privado, por la conservación y rehabilitación del patrimonio edificado en adobe de la región.

En el seguimiento del resurgido interés por la construcción con tierra, y en particular por la construcción en adobe, en el Departamento de Ingeniería Civil de la Universidad de Aveiro, un grupo de investigadores viene desarrollando en los últimos años un trabajo de estudio multidisciplinar, que se describe en el presente artículo, sobre las estructuras existentes de fábrica de adobe en la región de Aveiro con objetivo de, entre otros parámetros, caracterizar las propiedades mecánicas y de comportamiento estructural de construcciones de adobe. Particularmente, a través de la realización de análisis de caracterización dimensional, estudios de composición de adobes y de ensayos de caracterización mecánica de los bloques de adobe y de elementos de fábrica de adobe (en laboratorio e in-situ). Los resultados experimentales alcanzados con este trabajo sirven además para calibrar modelos numéricos que representen el comportamiento estructural, que podrán ser utilizados como herramienta de soporte en intervenciones de rehabilitación y refuerzo de construcciones existentes de adobe.

Asimismo, con el trabajo llevado a cabo se procuró contribuir a la creación de una base de resultados que posibilite, bien el apoyo a la ejecución de intervenciones de rehabilitación y/o refuerzo de estas estructuras, o bien el estudio de soluciones de mejora de sus características mecánicas que permitan corregir su comportamiento estructural y mejorar la seguridad, de las mismas sobre todo ante solicitaciones horizontales, como las inducidas por sismos.

\section{CARACTERIZACIÓN CONSTRUCTIVA}

Parte fundamental del trabajo realizado fue la toma de datos de campo de diferentes construcciones existentes en la región. La caracterización constructiva de los edificios
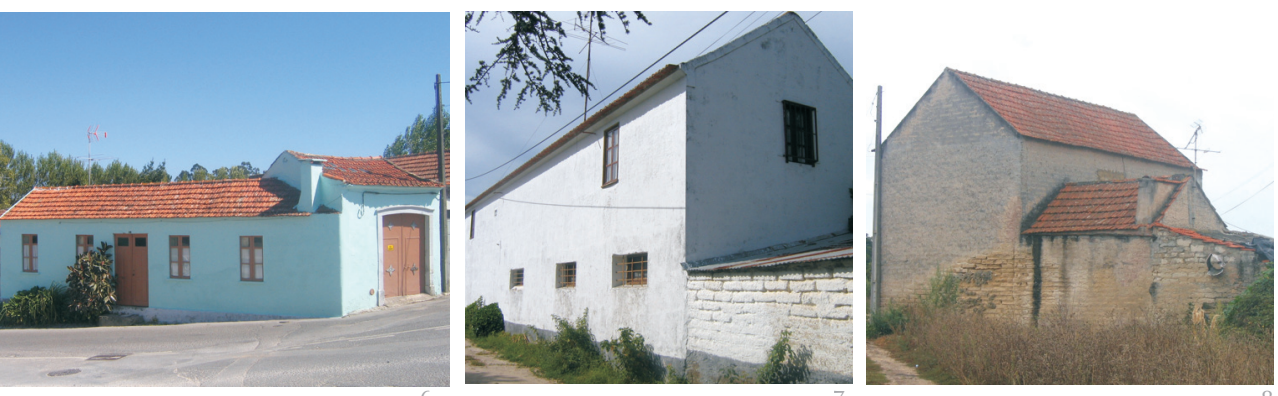

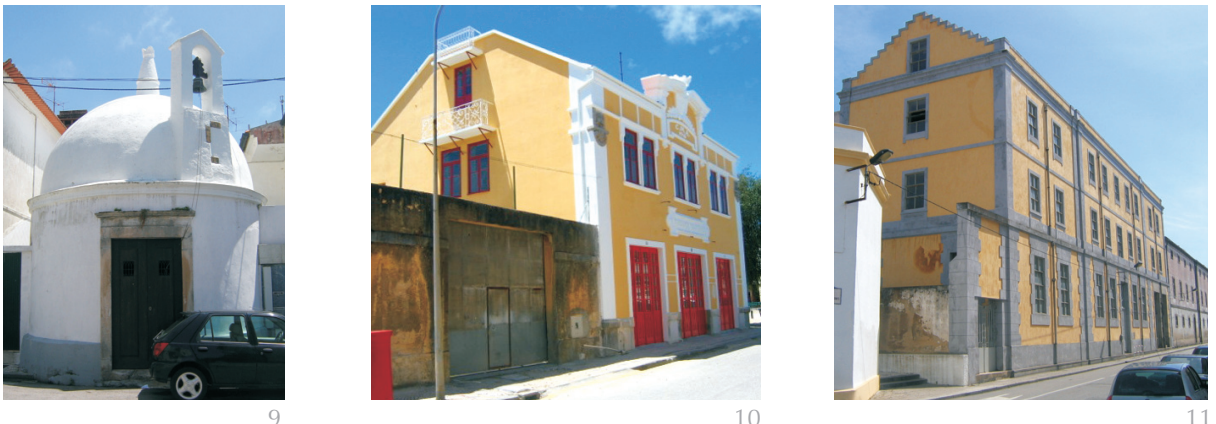

de adobe estudiados permitió constatar la gran diversidad tipológica y constructiva de fábricas de adobe existentes (Figura 1). Asimismo, se hace notar que la heterogeneidad detectada era considerable a distintos niveles, en el tamaño de los bloques y espesor de juntas de mortero, en su constitución y fabricación, en la configuración de las fábricas, etc., aspectos a los que se hace referencia con más detalle en el punto 3. Pese a ello, es posible diferenciar dos realidades bien distintas (edificios rurales vs. edificios urbanos, ver Figura 1). Asimismo, mientras que en zonas rurales, los edificios, de geometría principal en planta rectangular, presentan mayoritariamente una sola planta, en zonas urbanas éstos pueden presentar sensiblemente la misma geometría en planta, pero se verifica un aumento de número de plantas (en general dos, pero llegando hasta cuatro) y de la superficie en planta. También se verifica un mayor nivel cualitativo de albañilería y principalmente de los acabados, así como un mejor estado de conservación general de los edificios.

Se observa igualmente que, en algunos edificios, debido a la fuerte afección del agua sobre las propiedades del adobe (proveniente fundamentalmente de la humedad capilar, de una impermeabilización inadecuada y de la falta de conservación), la mejor solución tradicionalmente empleada consistió en la disposición de una cimentación de piedra, tratando de este modo de impedir el contacto directo entre los muros de adobe y el suelo (Figura 13-a). No obstante, se encuentran innumerables casos de cimientos de las fábricas realizados con adobe. En el caso particular de los pozos de agua, donde el contacto entre el agua y el adobe es constante, la solución de mejora empleada se basaba en la estabilización de los bloques y de los morteros empleados con mayores cantidades de cal, tratando de asegurar de este modo un mejor comportamiento y mayor durabilidad de los pozos en servicio (Figura 13-b).

En las aberturas se observa igualmente el empleo de dinteles de madera así como de arcos de distintos materiales (piedra, ladrillo y adobe), como se ejemplifica en las Figuras 14 a 16.

\section{CARACTERIZACIÓN DE LOS BLOQUES DE ADOBE}

\subsection{Diversidad tipológica}

La gran diversidad tipológica de adobes existentes en la región, en términos de composición, dimensiones, color, producción, etc. (ver Figuras 17-22), observada en los trabajos de campo realizados, no permite una clasificación inequívoca del adobe tipo de Aveiro. No obstante, en general, éstos están formados por suelo fundamentalmente arenoso (tierra), al que se añadió, durante su producción, con objeto de mejorar su comportamiento en servicio, un porcentaje considerable de cal. En este sentido, cabe señalar que, en general, las tierras empleadas en los adobes de la región presentaban estructura particular de tipo gravilla, con escasos vínculos de arciIla entre áridos por lo que el porcentaje de limos y arcillas era muy reducido frente a la predominancia casi exclusiva de arenas.

Las dimensiones más comunes de los bloques de adobe suelen variar en función de su uso particular. En general, en los edificios se empleaban bloques con dimensiones de referencia de $0,45 \times(0,30$ a 0,40$) \times$ $0,12 \mathrm{~m}$, mientras que en los muros de separación de parcelas agrícolas se empleaban bloques de $0,45 \times(0,20$ a 0,25) $\times 0,12 \mathrm{~m}$ (1).

\subsection{Caracterización granulométrica}

El abordaje experimental de caracterización del adobe de la región se inició con la caracterización granulométrica de los áridos retirados de muestras de adobes re-

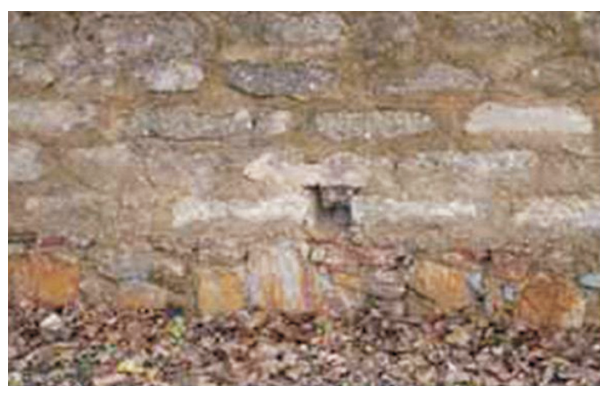

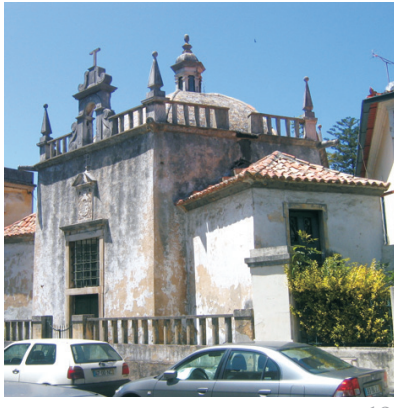

9-12. Ejemplos de construcciones de adobe en la región de Aveiro

13. Muro sobre cimentación de piedra e pozo de bloques de adobe

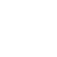



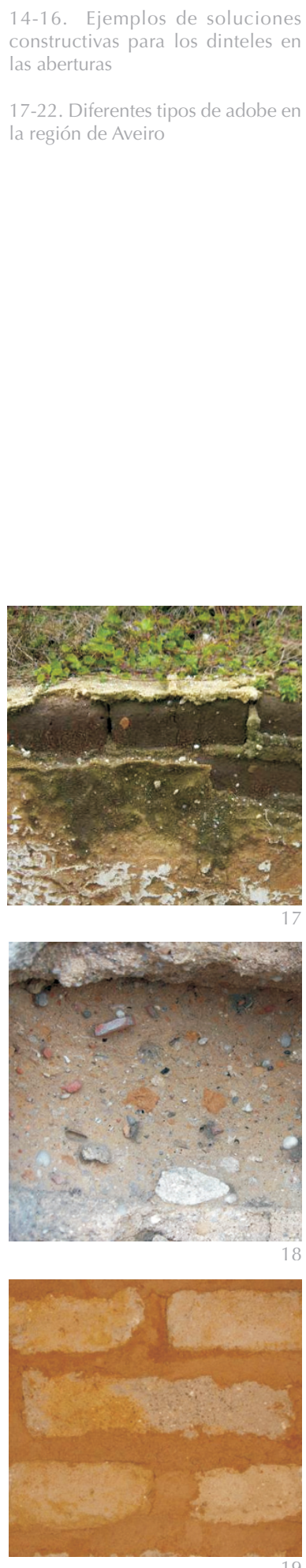
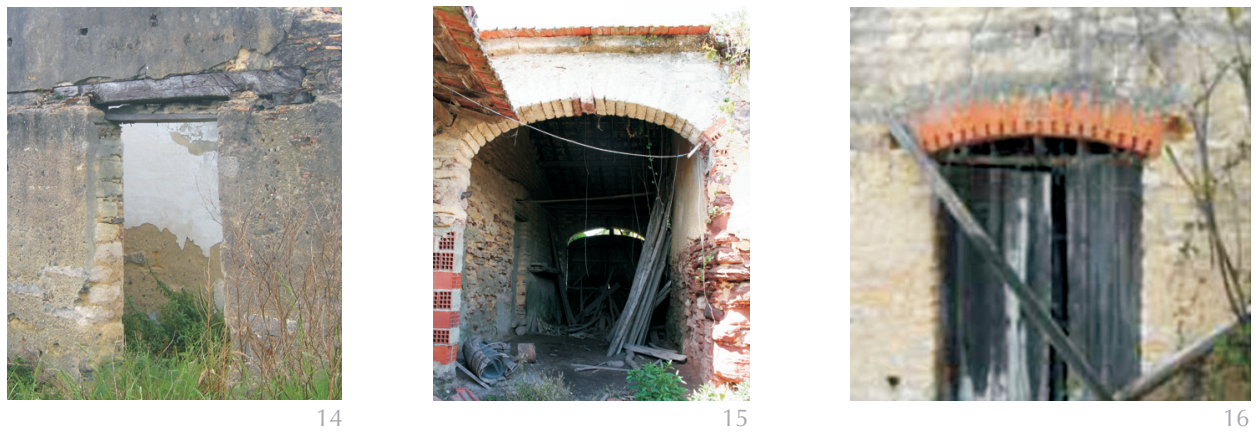

presentativos de la región, recogidas en los trabajos de campo realizados (2).

El ensayo realizado consistió resumidamente en la separación de una muestra de material, por medio de una serie de tamices, en diversas clases granulométricas (de granulometría decreciente). La dimensión de las aberturas y el número de tamices fueron seleccionados de acuerdo con el tipo de muestra (en este caso sobre todo de arena) y la precisión requerida.

Así, en relación con el procedimiento experimental llevado a cabo, se destacan las siguientes fases:

a) Se ejecuta la desagregación de los áridos de las muestras a ensayar.

b) Se establece la masa de cada una de las muestras que se pretende ensayar.

c) Se realiza el tamizado mecánico de los áridos, haciéndolos pasar por una serie de tamices de dimensiones decrecientes. Cabe señalar que los tamices utilizados en este estudio pertenecen a la serie ASTM.

d) Se pesan las fracciones retenidas en cada uno de los diferentes tamices, y se les asigna el porcentaje de masa total correspondiente.

e) Por último, se procede al trazado de las curvas de distribución granulométricas.

Dada la ya referida diversidad tipológica, fue seleccionado un conjunto de muestras representativas de los adobes de la región para la caracterización granulométrica de sus áridos. Así, para este fin se han seleccionado nueve muestras de muros de carga de edificios y doce muestras de muros de separación de parcelas agrícolas.

Dado que los adobes de la región están formados mayoritariamente por suelos arenosos, su caracterización granulométrica se realizó mediante el análisis por tamizado de los áridos de las muestras. Las curvas granulométricas así obtenidas confirman la naturaleza arenosa de los suelos tradicionalmente utilizados en la producción de los adobes, sin aptitud para usarse aisladamente, resultando, por ello, imprescindible la estabilización de los mismos con cal para su uso en la fábrica de adobe.

\subsection{Caracterización mecánica de los bloques}

Para la caracterización mecánica del adobe, se utilizaron probetas cilíndricas, extraídas por corte de los bloques del adobe recogidos de las construcciones muestreadas. Las probetas obtenidas tenían un diámetro de entre 60 y $95 \mathrm{~mm}$, y una altura correspondiente de dos diámetros. Así, fueron ensayados un total de 101 probetas; 83 a compresión simple y 18 a compresión diametral.

Los valores medios de resistencia a compresión simple obtenidos en los ensayos realizados, por construcción, se sitúan en el rango entre 0,53 MPa y 1,72 $\mathrm{MPa}$, siendo la correspondiente resistencia media de tracción de un $20 \%$ de la respectiva resistencia a compresión.
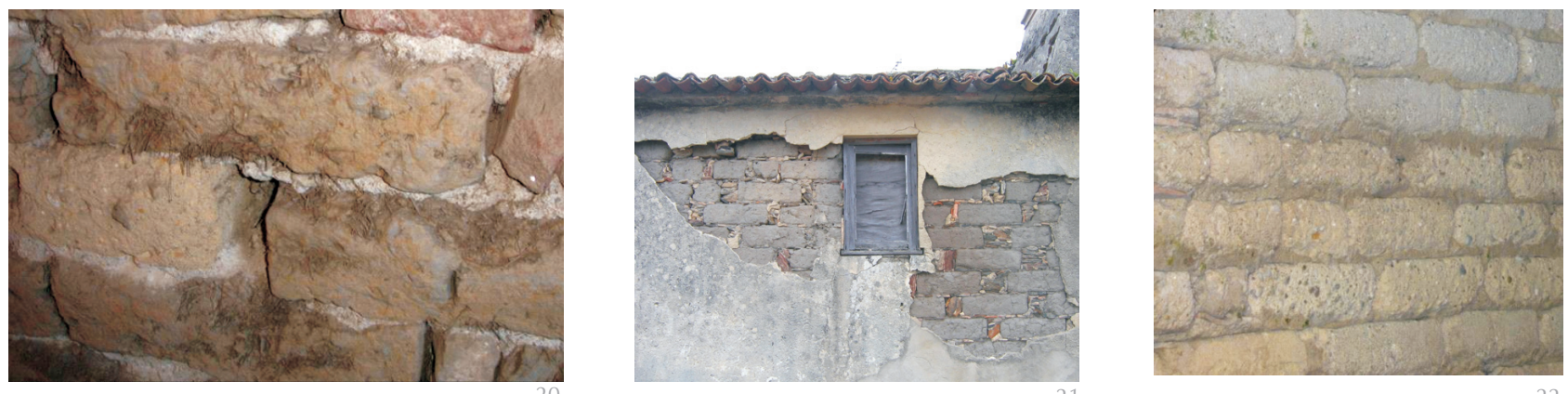
Desde el punto de vista de la granulometría de las muestras, se observó una clara tendencia para valores de resistencia mecánica superior en las muestras que presentan mayores fracciones de áridos de menores dimensiones.

\section{CARACTERIZACIÓN DEL COMPORTAMIENTO ESTRUCTURAL DE LA FÁBRICA DE ADOBE}

\subsection{Ensayos de laboratorio sobre muretes de adobe en escala reducida}

Para la caracterización mecánica de la fábrica de adobe representativa de la región, se llevaron a cabo inicialmente ensayos de compresión simple y diagonal sobre $13 \mathrm{mu}$ retes (Figuras 23-24) de dimensiones $17 \times$ $17 \times 10 \mathrm{~cm}$. Éstos fueron construidos con bloques recogidos de tres construcciones existentes (dos casas y un muro) durante el trabajo de campo. Así, los bloques originales recogidos, por condicionantes de dimensiones de la prensa usada para estos ensayos, fueron cortados, conservando la relación de escala típica de la fábrica en Aveiro, quedando sus dimensiones en $17 \times 10 \times 5 \mathrm{~cm}$. Teniendo como objeto mantener la composición de los morteros observados en el lugar de recogida de los bloques de adobe, se ha adoptado para la dosificación de los mismos, referida en volumen aparente, la proporción de una parte de cal hidratada, una de tierra ligeramente arcillosa y dos de arena. En su conjunto, el agregado tierra más arena, de acuerdo con el sistema unificado de clasificación de suelos, se corresponde con una arena. Cabe señalar que, el suelo empleado se recogió en el local referenciado por antiguos fabricantes de morteros y adobes. El espesor de las juntas fue de aproximadamente $1 \mathrm{~cm}$, manteniendo la misma reducción de escala impuesta a los bloques. Asimismo, los elementos de adobe fueron dispuestos en tres hiladas horizontales. Las hiladas superior e inferior estaban formadas por un solo bloque mientras que la hilada intermedia estaba formada por dos mitades separadas por una junta vertical de mortero.

Cabe señalar que los adobes utilizados en los muretes producidos presentaban una resistencia media a compresión simple, entre 0,77 y $1,72 \mathrm{MPa}$, mientras que en los ensayos de compresión del mortero usado en las juntas se obtuvo una resistencia a compresión media de 1,42 MPa.

Así, en los ensayos de compresión perpendiculares a las juntas de asentamiento sobre los muretes se obtuvieron valores de resistencia a compresión media entre 0,86 y $1,33 \mathrm{MPa}$. Los resultados pusieron en evi-
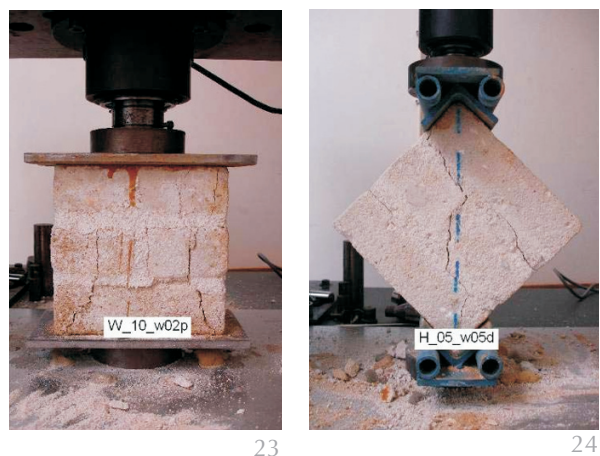

23-24. Ensayos de compresión perpendicular y diagonal sobre muretes de adobe

25-26. Resultados de los ensayos en compresión perpendicular y diagonal a las juntas de asentamiento

dencia una notable correlación entre la resistencia de compresión de los bloques de adobe y de los muretes correspondientes.

De acuerdo con los procedimientos de las recomendaciones RILEM (3), a través de las curvas tensión-deformación es posible estimar el valor de la tensión de rotura por corte así como el módulo de elasticidad transversal de cada murete. En los ensayos de compresión diagonal de los muretes se obtuvieron valores medios de tensión resistente de corte de entre 0,05 y 0,19 MPa. Se observó igualmente que para muretes construidos con bloques de adobe de menor resistencia
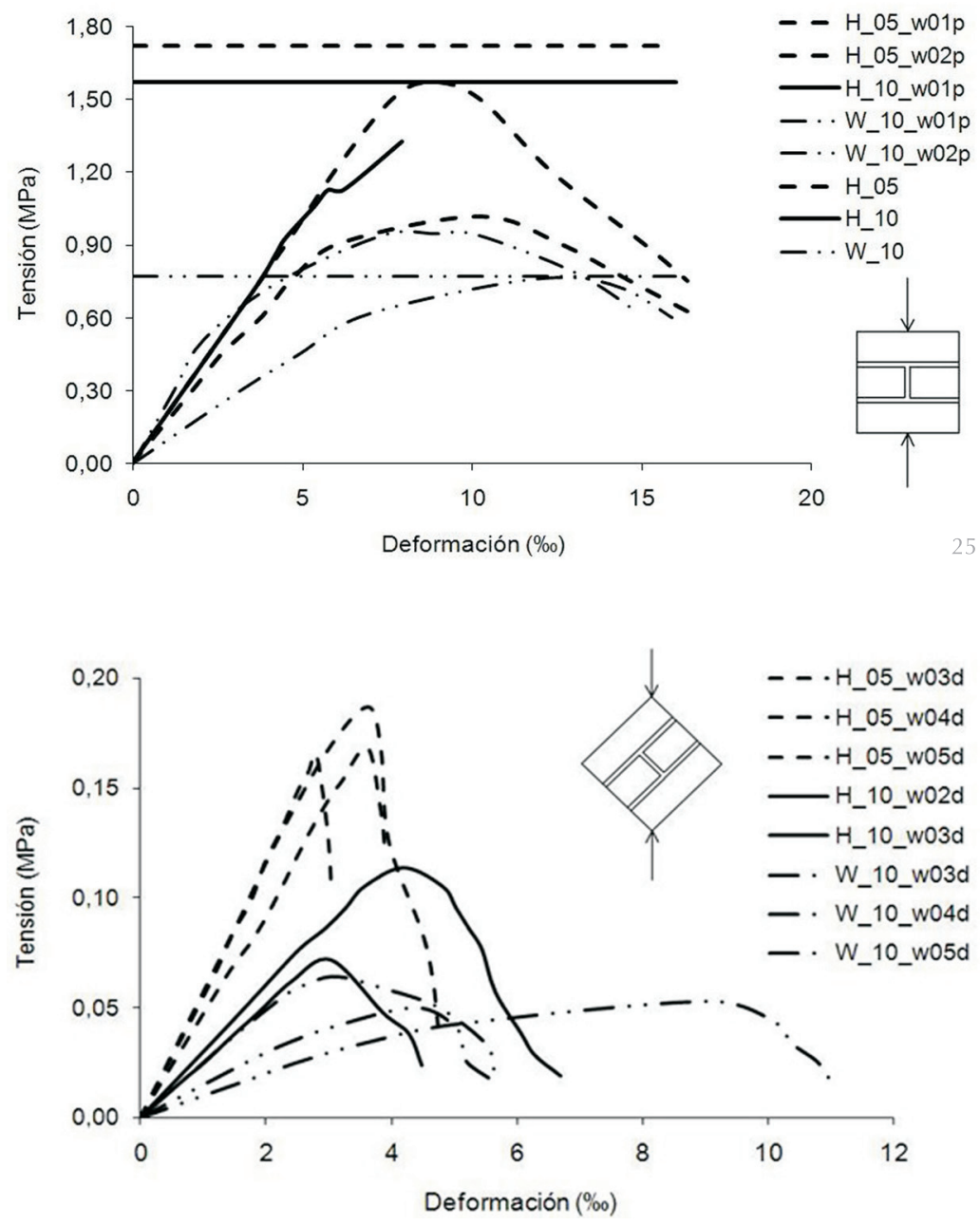
27-29. Construcción de la pared de adobe en el laboratorio a compresión se obtuvieron resistencias de corte inferiores. El módulo de elasticidad transversal y la resistencia al corte de los muretes ensayados es de aproximadamente $10 \%$ del módulo y resistencia a compresión correspondiente en los ensayos de compresión perpendicular a las juntas (4).

En la Figura 25-26 se presentan las curvas correspondientes a los ensayos de compresión perpendicular y diagonal realizados en los muretes. Téngase en cuenta que en el gráfico correspondiente al ensayo de compresión perpendicular a las juntas de asentamiento, se superpone todavía el valor de la tensión de rotura del adobe correspondiente utilizado.

\subsection{Ensayos de laboratorio sobre un panel modelo de pared de adobe}

\subsubsection{Construcción de la pared modelo y caracterización de los materiales}

En el laboratorio se construyó un panel modelo de pared en adobe, a escala real, cuya geometría en planta presentaba forma doble-T. Los bloques utilizados en su construcción fueron recogidos de un edificio objeto de demolición. Éstos presentan, en general, unas dimensiones de $45 \times 29 \times 12 \mathrm{~cm}$ y un peso volumétrico aparente de $16 \mathrm{kN} / \mathrm{m}^{3}$. El mortero utilizado en la construcción del panel de pared fue análogo al encontrado en el edificio demolido. La relación empleada fue de 1:1:2 (cal hidratada, tierra ligeramente arcillosa y arena). Como ya se ha indicado, el suelo empleado en la producción del mortero se recogió en el local referenciado por antiguos fabricantes de morteros y adobes siendo que, en su conjunto, el agregado tierra más arena, de acuerdo con el sistema unificado de clasificación de suelos, se corresponde con una arena.

La geometría en planta del panel de pared, doble $\mathrm{T}$, permite simular la influencia de la conexión de una pared a dos paños ortogonales, representando una situación común en las construcciones. Las dimensiones de la pared principal son 3,07 m (altura), 3,50 m (largo) y 0,29 m (ancho). La primera hilada de bloques se conectó a la cimentación con un mortero de cemento con el objetivo de impedir la rotura por deslizamiento de la base durante el ensayo (Figuras 27-29). Teniendo en cuenta que la superficie de la base del laboratorio sobre la que apoyaba la primera hilada de bloques de adobe era lisa, a efectos de rozamiento, no representando, por ello, las condiciones naturales de conexión entre la cimentación y la fábrica de una construcción corriente, con objeto de reproducir las condiciones de cimentación reales, se ha empleado un mortero de base cementicia.

En paralelo con los ensayos realizados sobre el panel de pared se ensayaron a flexión y compresión probetas prismáticas, de $16 \times 4 \times 4 \mathrm{~cm}$, del mortero elaborado para la construcción de la pared (juntas y revoque). Se obtuvo una tensión media de rotura en compresión de 0,67 $\mathrm{MPa}$ y una tensión media de rotura en flexión de aproximadamente el 35\% de este valor.

\subsubsection{Preparación de la campaña de ensayos sobre la pared e instrumentación}

La pared fue sometida a una fuerza horizontal cíclica de amplitud creciente, hasta su rotura. El ensayo fue controlado en términos de fuerza, la cual se aplicó para sucesivas amplitudes de diferentes series de ciclos de 30 kN, 50 kN y 60 kN. La cota de aplicación de la fuerza se situó a 2,50 m, medida desde la primera hilada de adobes del panel de pared mediante actuador hidráulico de 100 kN de capacidad. Para simular las cargas permanentes (peso propio de la estructura de las cubiertas y de sus revestimientos) y las sobrecargas existentes a que estas paredes están típicamente sometidas en las construcciones, se aplicó en la parte superior de la pared una carga vertical de $20 \mathrm{kN}$, uniformemente distribuida. El registro de la evolución de la respuesta de la pared a la aplicación de la fuerza horizontal cíclica se realizó con transductores de desplazamiento colocados en los puntos más representativos de su respuesta estructural.
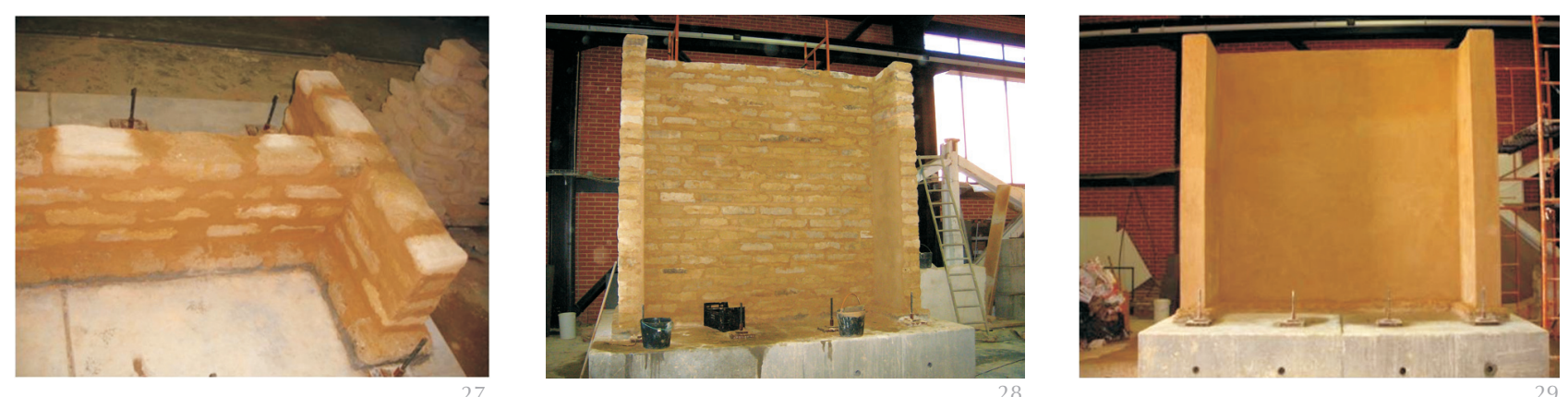


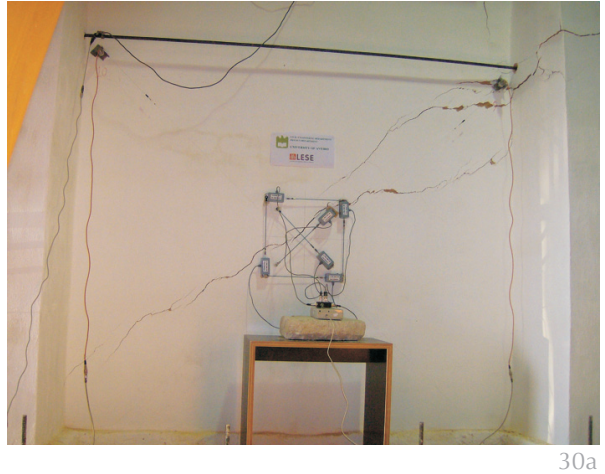

Con objeto de evaluar las propiedades dinámicas de la pared, particularmente las frecuencias naturales, se dispuso en su parte superior un sismógrafo que registró las aceleraciones provocadas por una excitación inducida entre ciclos de carga. Asimismo, al final de cada ciclo de fuerzas horizontales impuestas, se tomaba la primera frecuencia natural de la pared.

\subsubsection{Resultados de los ensayos realizados sobre el panel modelo de pared}

La prueba cíclica permitió caracterizar la capacidad resistente al corte de la pared, así como la máxima capacidad de deformación lateral, drift máximo, y el modo de rotura.

Para el cálculo de la tensión de corte sobre la pared se ha considerado como sección resistente la del plano del alma de la misma. Además, se ha considerado una distribución uniforme de tensiones a lo largo de este plano. En la figura 31 se puede observar la respuesta de la pared medida en la prueba cíclica, tensión de corte versus drift lateral de la pared.

Así, se observa que la capacidad lateral resistente de la pared es de aproximadamente $60 \mathrm{kPa}$, y se verifica para un drift lateral de aproximadamente $0,05 \%$. El drift máximo impuesto fue de $0,61 \%$, con una tensión corte instalada de aproximadamente $35 \mathrm{kPa}$. Esta resistencia residual de la pared corresponde a unos $60 \%$ de su capacidad máxima de resistencia al corte.

La pared sometida a la imposición de fuerzas horizontales cíclicas crecientes mostró una respuesta bastante linear hasta su resistencia máxima (Figura 30-a), y una vez alcanzada esta resistencia se verificó una rotura frágil, comportamiento típico de este tipo de elementos estructurales. A través de la Figura 30 se representa la evolución de los daños durante el ensayo. En la Figura 30-b se observa el aspecto final de la pared, posterior a la rotura.

De los ensayos dinámicos realizados al final de cada ciclo, se obtuvo la evolución

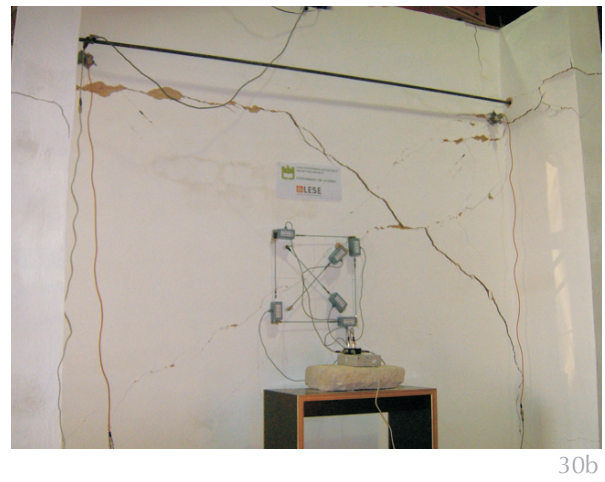

30a-30b. Evolución de los daños en la pared (5)

31. Tensión versus drift

32. Evolución de la primera frecuencia natural de la pared en función del máximo drift

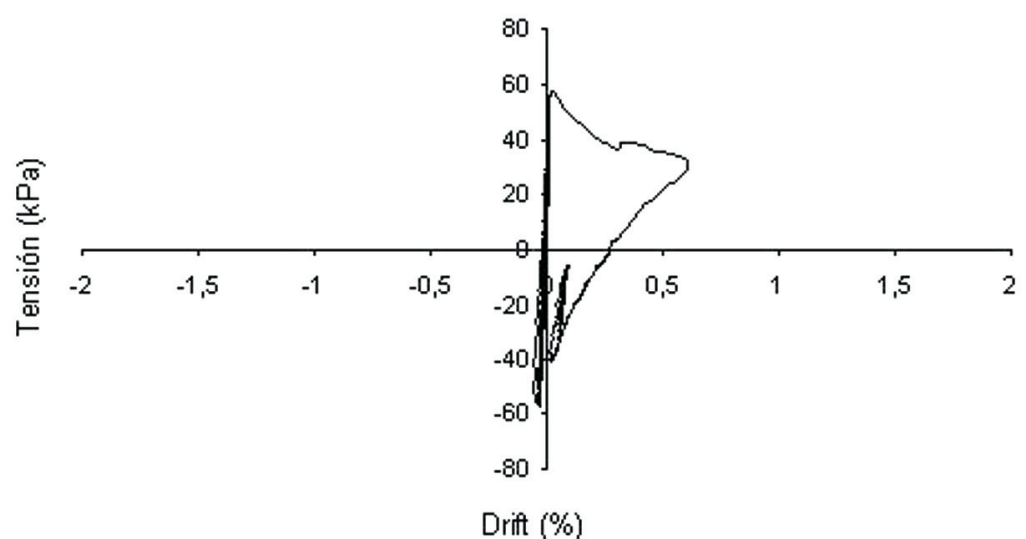

de la primera frecuencia natural de la pared en función del máximo desplazamiento impuesto (ver Figura 32).

\subsection{Solución de reparación y refuerzo de panel modelo de pared de adobe}

Una vez terminado el ensayo de caracterización del comportamiento de la pared original, se ideó y aplicó en ésta una solución de reparación y refuerzo. La solución ideada procuró garantizar la restitución a la pared de su rigidez y resistencia iniciales, mejorando además su desempeño, en términos de capacidad de deformación y ductilidad, frente a solicitaciones horizontales, como sean las inducidas por un sismo, y que garantizase, además, una buena compatibilidad entre los materiales utilizados y los originales. Se buscó igualmente una solución económica y fácil de aplicar.

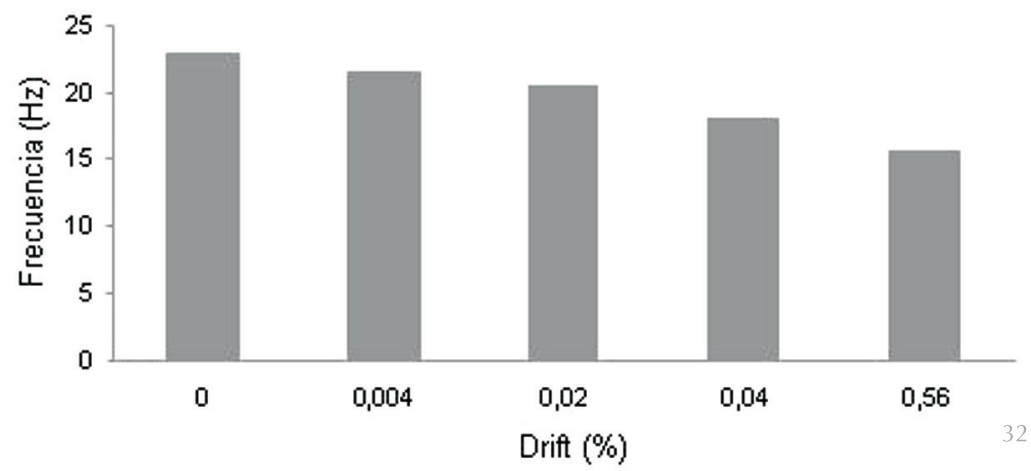


33. Eficiencia del sellado de fisuras (recuperación de la resistencia inicial, \%)
Asimismo, como solución de reparación se ha optado por la aplicación a presión de un mortero formado únicamente por un ligante de cal hidráulica para el sellado de fisuras. En el refuerzo se empleó una red sintética, estirada, envolviendo toda la pared, con objeto de mejorar su capacidad de deformación y ductilidad. La red seleccionada para esta solución presenta como principales características: el reducido grosor de la fibra $(0,8 \times 0,6 \mathrm{~mm})$, un peso específico de $55,11 \mathrm{~g} / \mathrm{m}^{2}$, una buena flexibilidad, permitiendo un fácil ajuste a las irregularidades de los bloques de adobe, una superficie de los filamentos no totalmente lisa, que facilita la adherencia al mortero, y una resistencia mecánica adecuada, que evite su rotura por tracción cuando sea solicitada la pared reforzada. Al no ser facilitada por el fabricante la ficha técnica de la red, se llevaron a cabo algunos ensayos de tracción (punto 4.3.1.3) para obtener más información sobre de sus propiedades.

\subsubsection{Caracterización mecánica de los materiales empleados en la reparación y refuerzo de la pared}

\subsubsection{Caracterización del mortero de reparación}

Al igual que durante la construcción de la pared, en la fase de reparación y refuerzo de la misma, fueron confeccionadas probetas con objeto de caracterizar mecánicamente el mortero empleado en dichas labores.

Las probetas obtenidas del mortero utilizado en la inyección de las fisuras (reparación de la pared) alcanzaron una resistencia media a compresión de 3,77 MPa, mientras que su resistencia a tracción fue de aproximadamente el $30 \%$ del valor de resistencia a compresión. En los ensayos de caracterización mecánica del mortero empleado en el revestimiento de la pared, en el cual quedó embebida la red sintética (refuerzo), se obtuvo una resistencia a compresión media de 0,61 MPa y una resistencia a tracción media de 0,20 MPa, que corresponde aproximadamente al 30\% de la resistencia a compresión obtenida.

\subsubsection{Ensayos de flexión de los bloques} de adobe

Con el objetivo de analizar la solución más eficaz de sellado de las fisuras existentes en la pared, se han desarrollado una serie de ensayos de flexión sobre bloques de adobe. Así, fueron inicialmente ensayados a flexión una serie de bloques de adobe, donde se obtuvo una tensión media de rotura por tracción de 0,54 MPa. Con las mitades de bloques obtenidas de los ensayos de flexión, se ha procedido a su pegado con diferentes tipos de ligantes, específicamente: (i) cal hidráulica; (ii) cal hidratada; e (iii) mezcla de cal hidráulica y cal hidratada, en partes iguales. Después de reparados los bloques con distintas soluciones, éstos fueron de nuevo ensayados a flexión. Tal y como se deduce del gráfico de la figura 33, el mortero de cal hidráulica permite un pegado más eficiente de los bloques de adobe, pudiéndose alcanzar con esta solución una recuperación de la resistencia en torno a un $65 \%$.

\subsubsection{Ensayo de tracción de la red sintética de refuerzo}

Con el fin de caracterizar la red empleada en el refuerzo de la pared, se llevaron a cabo ensayos de tracción sobre la misma. El ensayo de tracción se realizó a una velocidad de desplazamientos impuestos de $3 \mathrm{~mm} / \mathrm{min}$ y con una temperatura ambiente de $22{ }^{\circ} \mathrm{C}$.

Los resultados en términos de la curva tensión-deformación de la red se representan en la figura 34. Se obtuvo una tensión media de rotura a tracción de 9 MPa para una deformación longitudinal correspondiente del $18 \%$. Para la red se observó un modo de rotura frágil de las fibras.

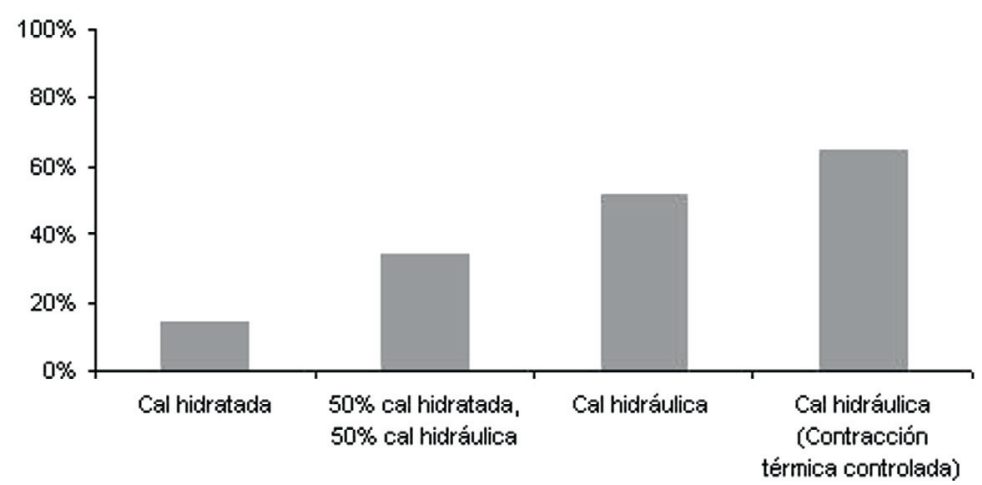



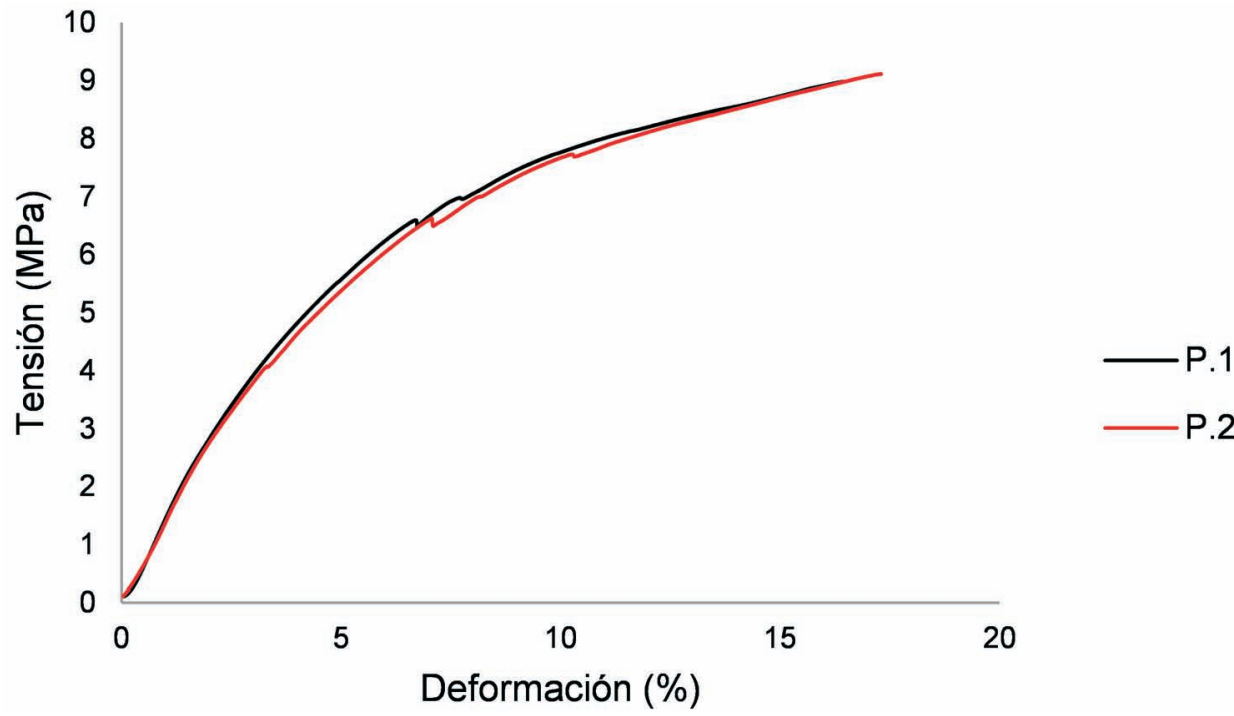

4.3.2. Trabajos de reparación y refuerzo del panel modelo de pared de adobe

\subsubsection{Reparación de los daños resultantes del ensayo de caracterización}

Concluidos los ensayos descritos en el punto 4.2 , que terminaron con la rotura del panel de pared, se hacía necesario reparar los daños existentes para posteriormente poder aplicar la técnica de refuerzo propuesta. La metodología empleada consistió en el relleno de las fisuras existentes por inyección bajo presión de un mortero de cal hidráulica. Las principales fases de empleo de esta solución fueron:

- Apertura, mediante taladro, de agujeros espaciados de unos $20 \mathrm{~cm}$ a lo largo del trazado de las fisuras;

- Inserción en los agujeros de tubos de plástico con un diámetro interior de 12 $\mathrm{mm}$ hasta el interior de las fisuras (Figura 36-a);

- Sellado exterior de las fisuras con mortero de cal hidráulica (Figura 36-b) que imposibilite la salida del mortero a inyectar posteriormente;

- Inyección de las fisuras con agua, con el fin de minimizar los efectos de la retracción del mortero de inyección;

- Inyección de mortero de cal hidráulica a presión (Figura 36-c).

\subsubsection{Aplicación de la solución de refuerzo sísmico}

Una vez reparado el panel de pared, se pasó a la fase de refuerzo sísmico del mismo. Se procedió, inicialmente, a la retirada del revoque primitivo de la pared para posterior aplicación de un nuevo revoque con la red sintética en su interior. Así, las principales tareas de la fase de refuerzo de la pared fueron (Figura 37):

- Aplicación de la red sintética envolviendo todo el panel de pared, debidamente estirada y anclada a la base;

- Fijación de la red con angulares de PVC a la base y a todos los vértices cóncavos de la pared. Los angulares de los vértices cóncavos se encontraban "cosidos" a un perfil de PVC laminado en el trasdós de la pared con un hilo de nylon;

- Clavado de la red a la pared con tacos/ clavos de plástico (de 6,0 cm de diámetro de la cabeza y $0,7 \mathrm{~cm}$ del perno, que penetran en la pared unos $6,5 \mathrm{~cm}$ ) distribuidos en una malla aproximadamente cuadrada, de 0,5 m de lado;

- Aplicación del nuevo revoque, con características idénticas al revoque original, envolviendo la red sintética.

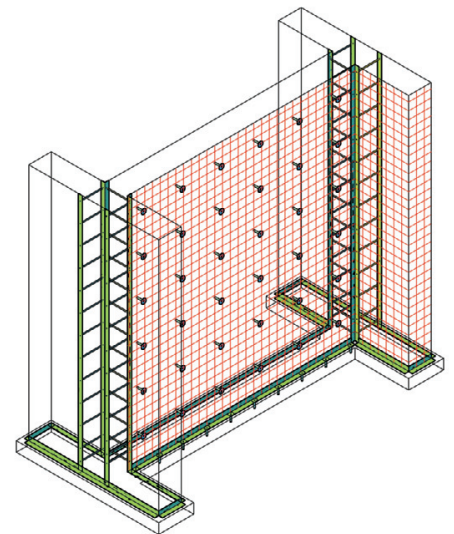

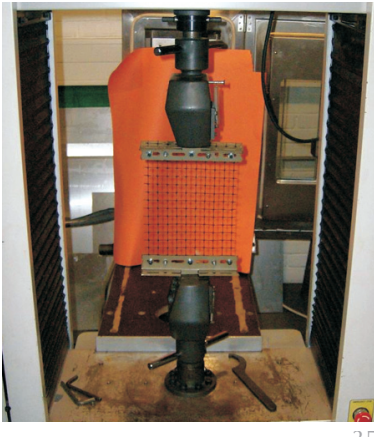
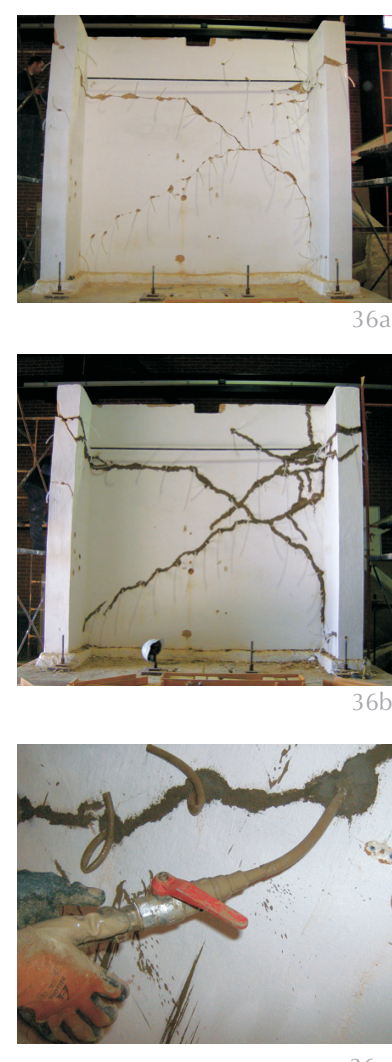

34-35. Ensayo de tracción de la red de refuerzo y resultados obtenidos 36. Trabajos de reparación: (a) tubos de inyección; (b) sellado de las fisuras; y, (c) inyección del mortero de cal hidráulica

37. Solución de refuerzo adoptada

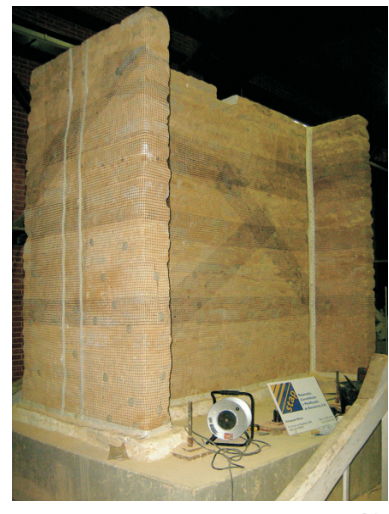


38. Resultados del ensayo cíclico de la pared reforzada: (a) curva tensión versus drift; (b) evolución de la energía disipada
Cabe señalar que las fases de la reparación y refuerzo de la pared se describen con más detalle en (6).

\subsubsection{Resultados de los ensayos cíclicos sobre el panel de pared reforzado}

En esta segunda fase de ensayos sobre la pared reforzada se ha tratado de mantener el mismo procedimiento empleado en los ensayos realizados anteriormente sobre la pared en su estado original. El punto de aplicación de la fuerza y la posición de los transductores electrónicos se mantuvieron sin cambios.

Tabla 1

Valores de drift impuestos a la pared reforzada durante la prueba cíclica

\begin{tabular}{|l|c|c|c|c|c|c|c|c|}
\hline \multicolumn{2}{|c|}{ Drift (\%) } \\
\hline $0,005 \%$ & $0,01 \%$ & $0,02 \%$ & $0,05 \%$ & $0,1 \%$ & $0,2 \%$ & $0,5 \%$ & $1,0 \%$ & $1,5 \%$ \\
\hline
\end{tabular}

Para la prueba de la pared reforzada se han empleado ciclos impuestos de desplazamiento correspondientes a niveles de drift que se presentan en la Tabla 1. Al igual que en el ensayo de la pared original, los desplazamientos horizontales de la pared y deformaciones en puntos críticos fueron medidos por potenciómetros de hilo y por LVDT's. En este ensayo de la pared reforzada también se utilizó un sistema de alta resolución, basado en técnicas de radar, para caracterizar la evolución del estado interno de daño de la pared de adobe (7). Los resultados alcanzados con estas medidas permitieron identificar con rigor el momento en el que se iniciaba la formación de fisuras y su evolución.

De la respuesta dada por la pared reforzada ante los desplazamientos horizontales cíclicos impuestos (Figura 38) se recabaron los principales resultados, que se describen a continuación:

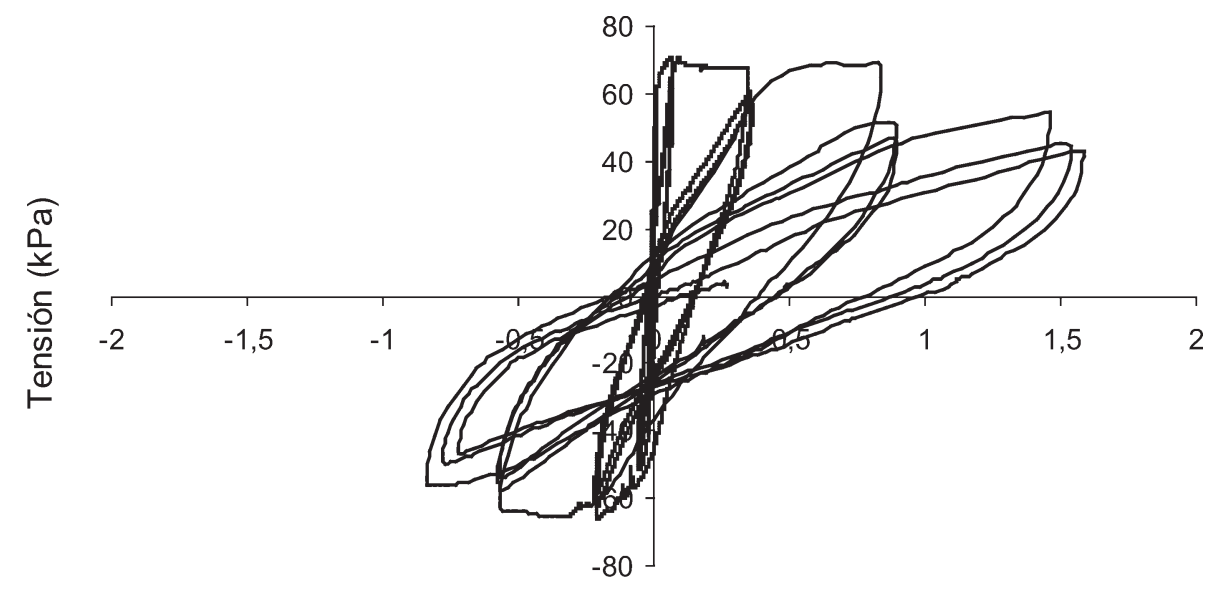

Drift $(\%)$

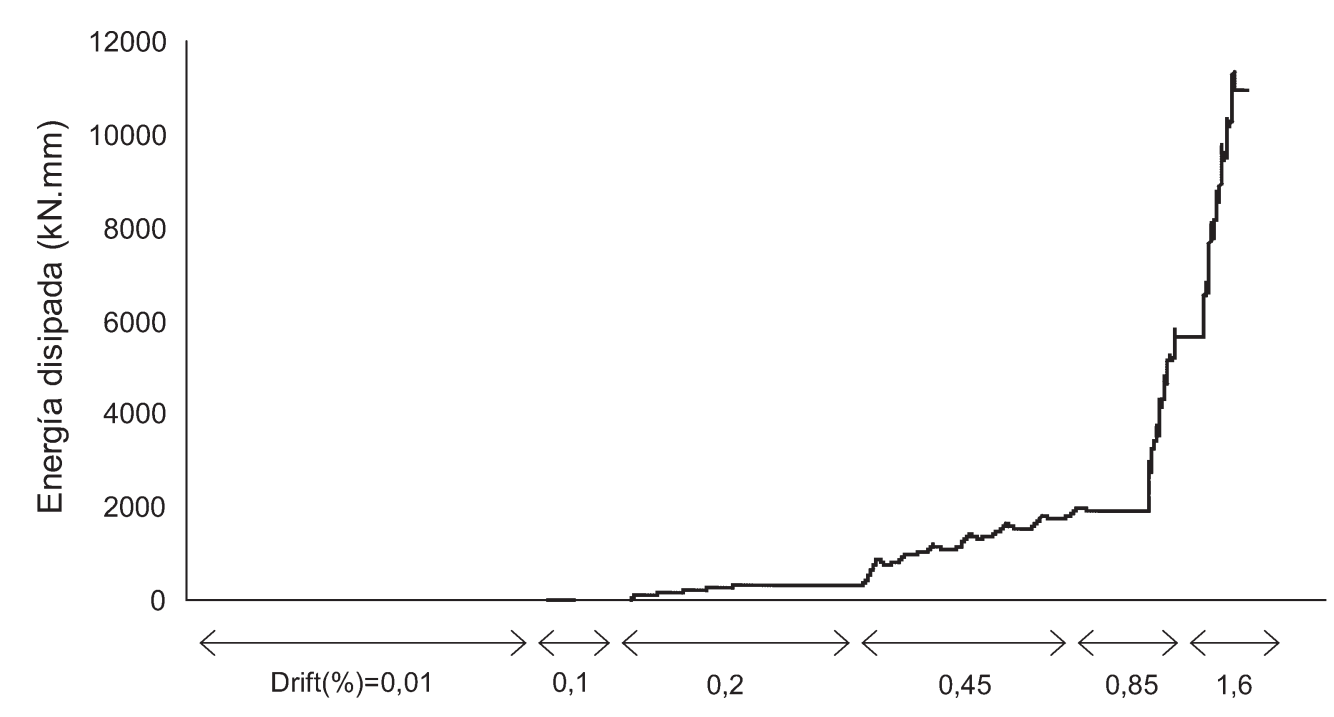


- En los primeros ciclos de desplazamientos impuestos se observa una respuesta casi lineal de la pared;

- Aún en los primeros ciclos de desplazamientos impuestos se verifica que la disipación de energía fue prácticamente nula, asociada a la ausencia de daños observados;

- La tensión máxima de corte aplicada en la pared fue de aproximadamente $67 \mathrm{kPa}$, para un drift correspondiente de $0,1 \%$;

- La resistencia residual obtenida fue de $45 \mathrm{kPa}$ (aproximadamente $70 \%$ de su resistencia máxima);

- La deformación máxima impuesta corresponde a un drift de 1,6\%;

- Entre los ciclos de drift de 0,1\% y $0,85 \%$, se observa una disipación de energía cercana a 1/5 de la energía total disipada durante todo el ensayo. Esta disipación de energía está asociada al inicio de la formación de daños en la pared;

- En los últimos ciclos (a partir de 0,85\% de drift), los daños en la pared crecieron de forma exponencial, hecho que se puede comprobar en el gráfico de evolución de la energía disipada. Así se estima que $4 / 5$ de la energía total hayan sido disipados en estos ciclos.

Con los datos recabados durante el ensayo cíclico se ha podido comprobar que el inicio del agrietamiento ocurrió para un nivel de drift impuesto de aproximadamente 0,07\%. En el ciclo siguiente se observó un gran incremento de las fisuras ya existentes, observándose además la formación de nuevas fisuras. En paralelo con el incremento de amplitud de desplazamientos se ha ido observando un despegue del revoque en el área ya agrietada, que se iba acentuando según el desplazamiento impuesto aumentaba. En las Figuras 39 a 42 se observa la evolución de los daños en la pared en el transcurso del ensayo.

Para averiguar la eficiencia de la solución de refuerzo estudiada, se trazó la curva tensión-drift (Figura 43), comparativa entre los dos ensayos realizados en la pared, estado original y reforzado.

De la observación del gráfico se verifica la eficacia del refuerzo en la mejoría de la respuesta de la pared. Así, del grafico de la Figura 43 se concluye lo siguiente:

- La rigidez del panel de pared en su estado original, así como una vez reforzado es prácticamente igual;
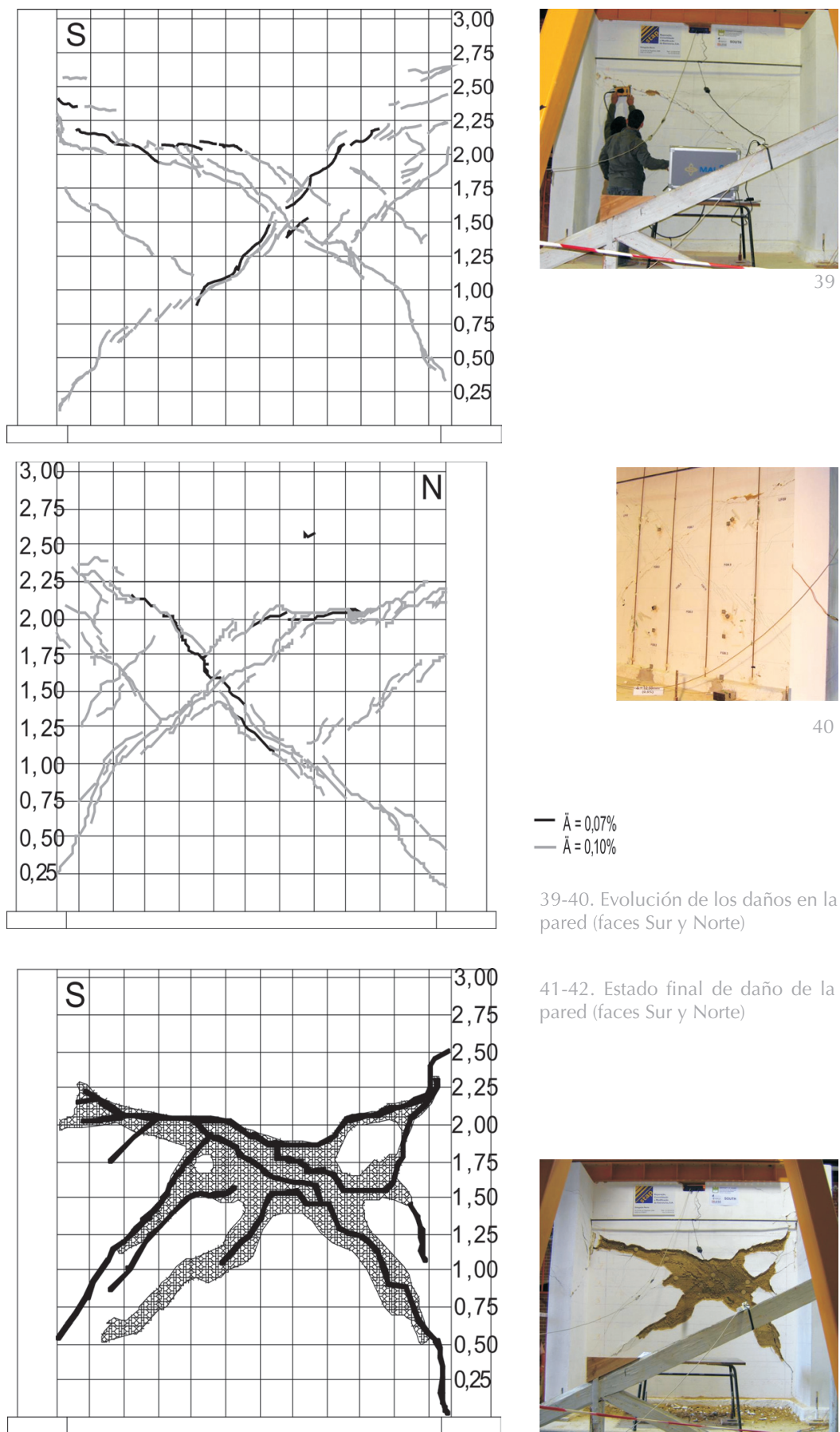

pared (faces Sur y Norte)

41-42. Estado final de daño de la pared (faces Sur y Norte)
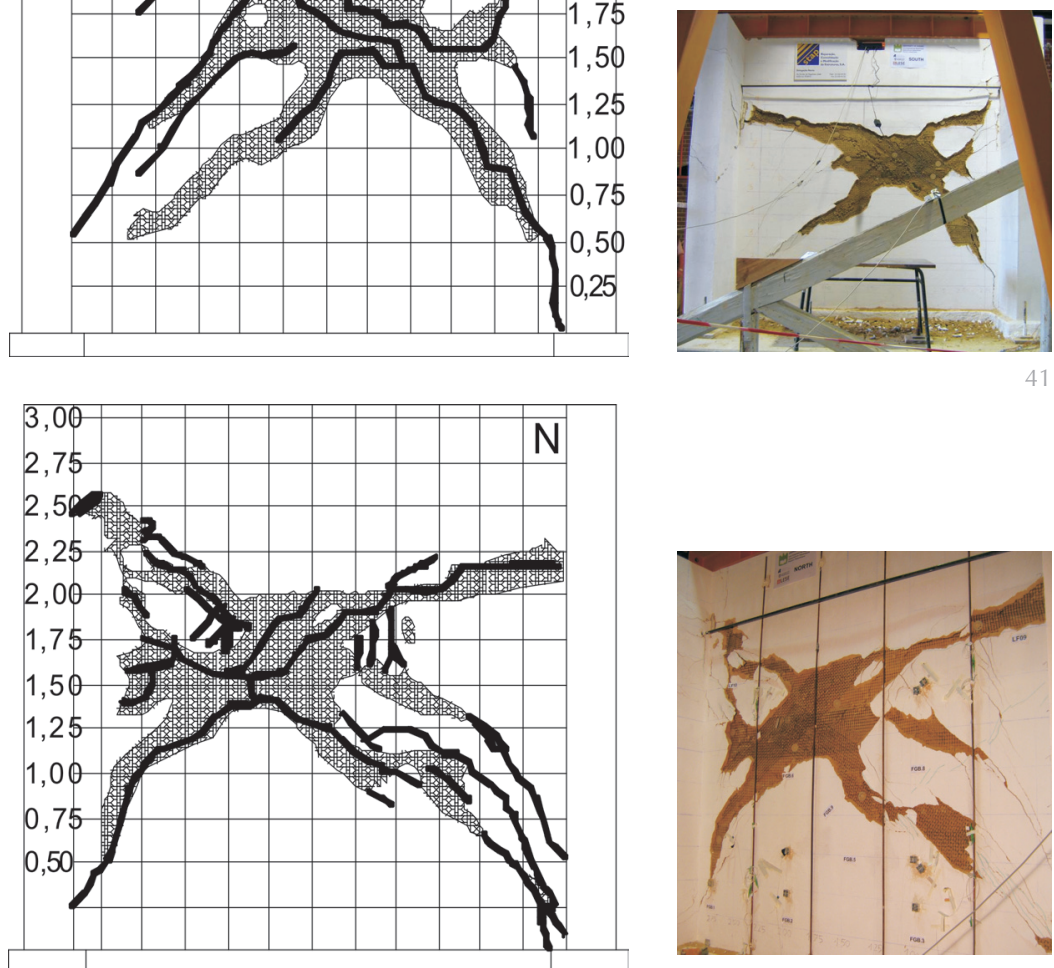


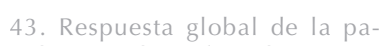
red (original y reforzada): curvas tensión-drift

44. Evolución de la primera frecuencia propia de la pared (original y reforzada) en función del drift máximo impuesto

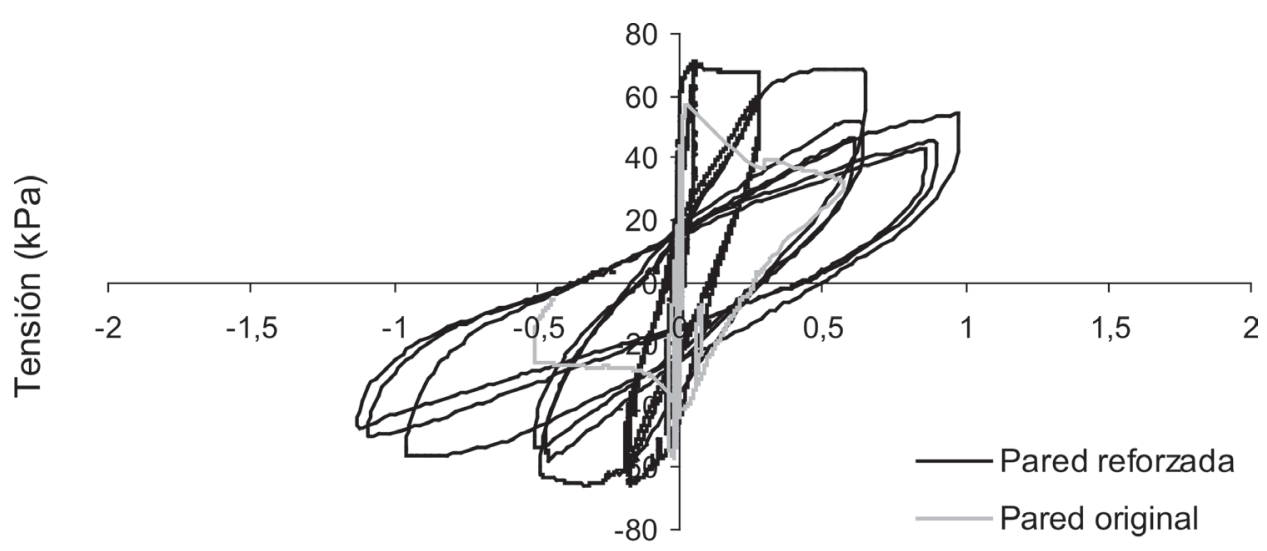

Drift $(\%)$

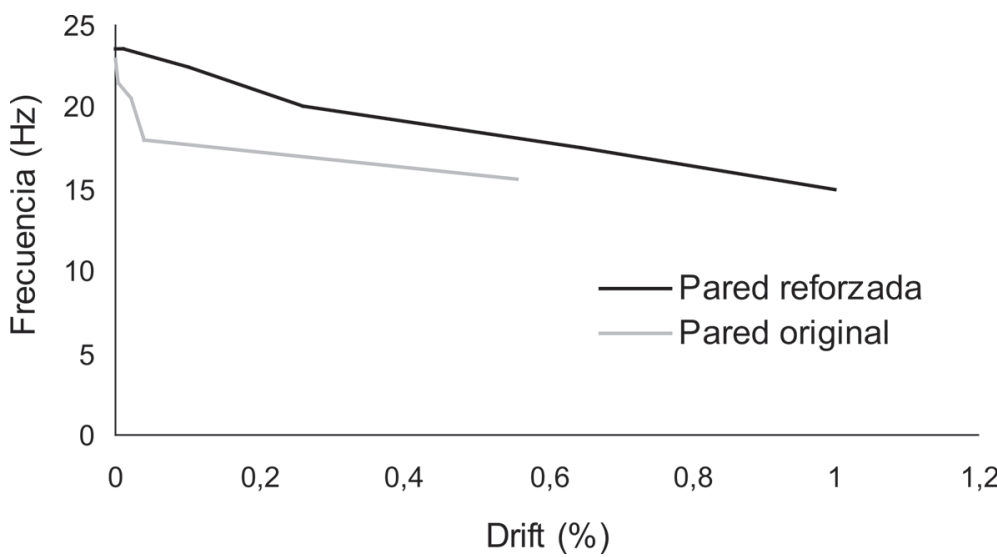

- La capacidad máxima resistente de la pared a corte ha experimentado un incremento de aproximadamente un $20 \%$, con las intervenciones de reparación y refuerzo;

- El drift máximo impuesto en la pared reforzada es cerca del doble del impuesto en la pared original, comprobándose así la mejoría en términos de capacidad de deformación;

- La fragilidad de la pared con el refuerzo adoptado disminuyó substancialmente. Para ciclos repetidos se observó una baja menor en la resistencia de la pared reforzada, después de ultrapasado el valor máximo de la resistencia. Así, la ductilidad y la capacidad de disipación de energía de la pared reforzada se ven largamente mejoradas;

- La solución de refuerzo empleada en la pared de adobe ha demostrado poseer una elevada eficacia en el refuerzo sísmico de la misma.
4.3.4. Resultados comparativos de los ensayos dinámicos del panel de pared original y del reforzado

Con objeto de evaluar la recuperación de rigidez alcanzada con la reparación de la pared y analizar su evolución durante el ensayo cíclico, se realizaron varias lecturas de las frecuencias propias de la pared (Fig. 44). El análisis comparativo de la evolución de dichas frecuencias para el ensayo cíclico sobre la pared original y reforzada pone en evidencia la diferencia observada en el comportamiento de la pared, cuando reforzada.

De la observación del punto inicial de las curvas de evolución de la primera frecuencia propia de la pared, se verifica que la rehabilitación restableció aproximadamente la rigidez inicial observada en la pared original $(22,95 \mathrm{~Hz}$ para la pared en su estado original y $23,00 \mathrm{~Hz}$ después de la reparación y refuerzo). En el gráfico de la Figura 44 se observa que, para la pared reforzada la disminución de rigidez, y por lo tanto de 

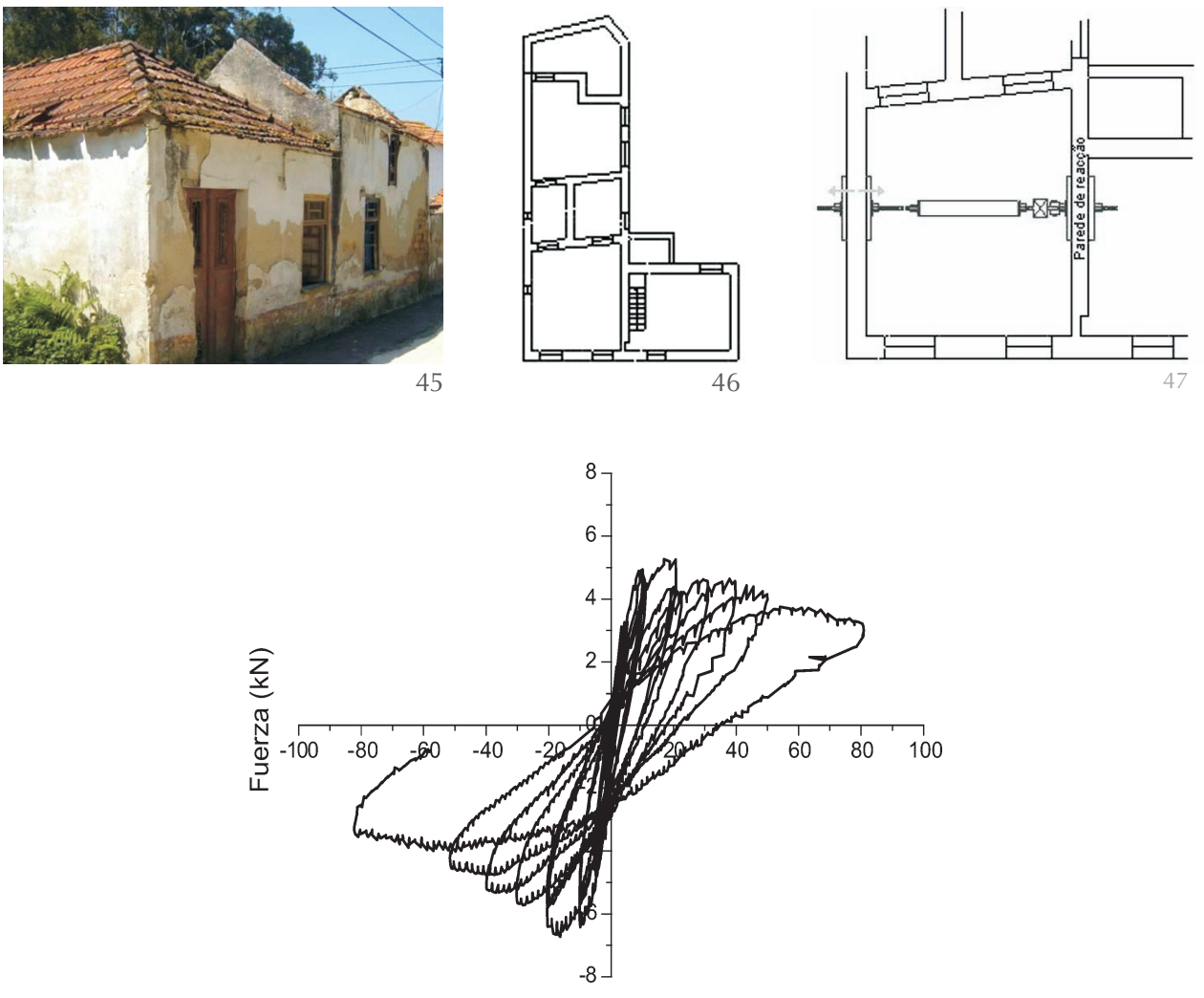

Desplazamiento $(\mathrm{mm})$

la frecuencia natural, con el aumento del nivel de exigencias de deformaciones es más suave que para la pared no reforzada (original). Este hecho está asociado a la mayor fragilidad de la pared original. También el análisis de la evolución de las frecuencias mostró que el refuerzo aplicado benefició significativamente el comportamiento y desempeño de la pared ante cargas cíclicas horizontales.

\subsection{Ensayos in-situ en construcciones existentes de adobe}

\subsubsection{Ensayos en una vivienda de un piso}

Con el fin de caracterizar el comportamiento mecánico in-situ de edificios existentes, se llevó a cabo una campaña de ensayos en una vivienda ubicada en una zona rural de Aveiro (Figura 45-47), con solicitaciones cíclicas horizontales (como las inducidas por los sismos). La vivienda en cuestión era un edificio con más de 80 años, que se encontraba abandonado y presentaba un estado de patología muy acentuado.

Se realizó un ensayo cíclico con imposición de una fuerza concentrada horizontal cíclica, en paredes directamente opuestas, en la dirección correspondiente para fuera del plano de las paredes, aplicadas a una cota de $+2,33$ m desde el suelo, utilizando un sistema de ensayo detalladamente descrito en (8).
Durante la aplicación de las fuerzas horizontales, se midieron los desplazamientos en puntos representativos de la respuesta de la estructura. En la Figura 48 se observa el gráfico que representa la evolución de la fuerza aplicada con el correspondiente desplazamiento horizontal de la pared en el punto de aplicación de la misma.

Los resultados obtenidos (Figura 48) mostraron que la pared se comportó de forma ligeramente asimétrica. Se obtuvo una resistencia máxima de 6,8 y $5 \mathrm{kN}$ en las direcciones correspondientes de aproximación y distanciamiento de las paredes ensayadas. Justo después de la aplicación de la fuerza máxima se observó un acusado daño en la pared y una pérdida abrupta de resistencia. Los daños se iniciaron con el despegue entre paños de pared en su conexión. El comportamiento observado es típico de muros de fábrica solicitados fuera de su plano, regulados por la inestabilidad geométrica de las paredes.

El límite del comportamiento elástico de la pared se observó para un drift de 0,4\%, y para la resistencia máxima de la pared de aproximadamente $0,8 \%$. El drift máximo impuesto en el ensayo fue de $3,8 \%$.

\subsubsection{Campaña de ensayos en una pared}

Se llevaron a cabo igualmente ensayos de caracterización estructural in-situ en una pared de una construcción en ruina, situa- 
49. Ejemplos de fichas de inspección y evaluación: a) nivel 2; b) nivel 3

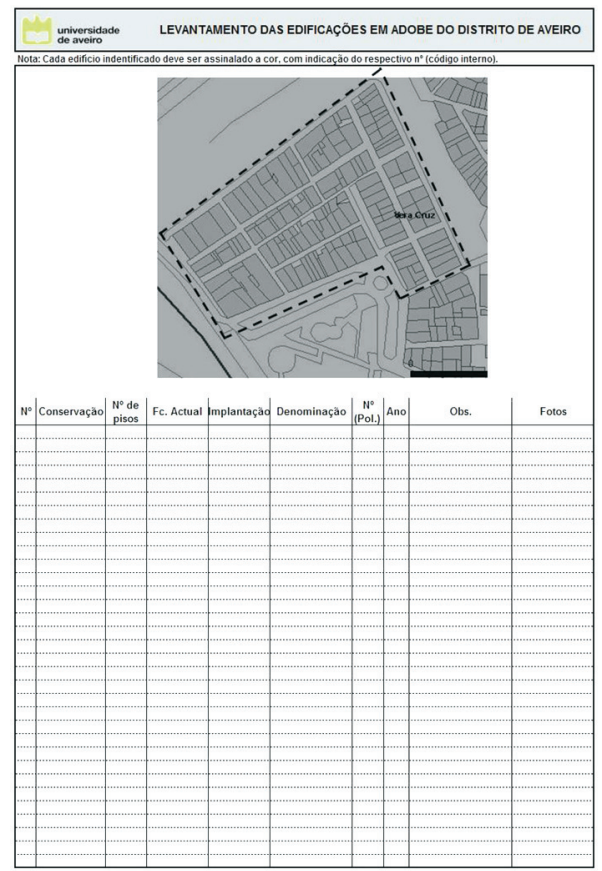

49 a
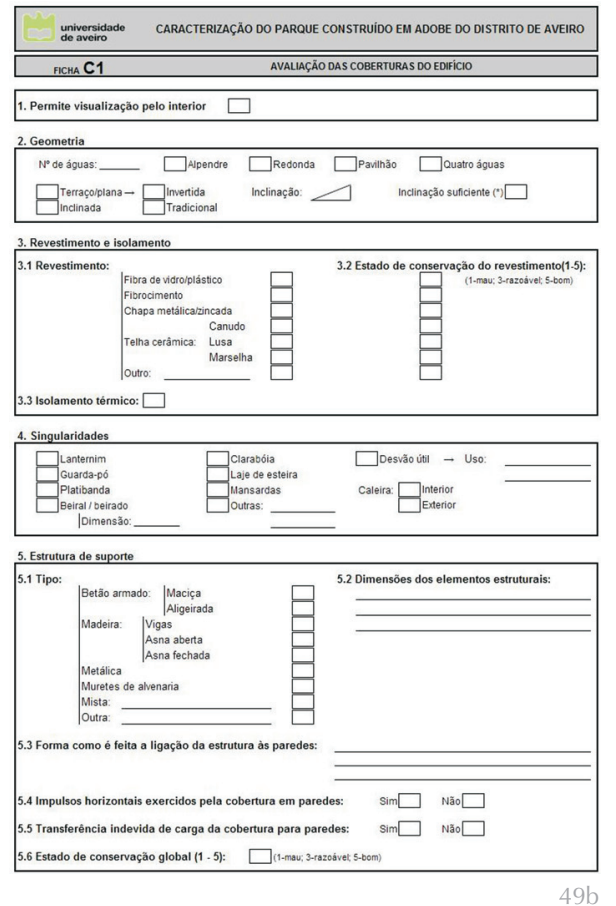

da en la localidad de Fermelã, Estarreja, en el distrito de Aveiro. Estos ensayos tuvieron como objetivo valorar y comparar, en condiciones reales de funcionamiento estructural, el comportamiento de paredes en el plano y fuera de su plano. Así, se realizaron ensayos cíclicos en dos fases. Primero con aplicación de fuerzas horizontales de amplitudes crecientes en el plano de la pared hasta alcanzar su resistencia máxima, y luego fuera del plano de la pared hasta su colapso.

En el ensayo fuera del plano de la pared se produjo un desplazamiento máximo de $16 \mathrm{~mm}$, correspondiente a un drift de 0,95\%, cuando se produjo el colapso de la pared. La comparación de resultados de los ensayos, en el plano y fuera del plano, pone de manifiesto valores de drift semejantes para fuerzas 15 veces inferiores en los ensayos fuera del plano. La descripción más detallada de estos ensayos y de sus resultados se presenta en (9).

\section{CONCLUSIONES Y COMENTARIOS FINALES}

En la primera parte del artículo se presenta la caracterización tipológica y constructiva de los edificios de adobe que se encuentran tanto en el casco urbano, como en las zonas rurales de la región de Aveiro, en la cual esta técnica constructiva se desarrolló con más expresión en Portugal. En los trabajos de campo realizados, se observaron por una parte edificios rurales con geometría básicamente rectangular y de una sola planta y por otra en el casco antiguo de la ciudad de Aveiro, edificios en los que pese a que presentan igualmente geometría en planta rectangular, resulta reseñable el aumento de plantas en los mismos, un mayor nivel cualitativo de albañilería y acabados y generalmente un mejor estado de conservación de éstos.

Además, los trabajos de campo llevados a cabo permitieron caracterizar mecánicamente el adobe de la región. Así, pese a la gran diversidad de adobes que se encontraron, es posible recabar algunas conclusiones de la investigación desarrollada:

- La resistencia media a compresión simple obtenida de los bloques de adobe muestreados varía entre 0,5 a 1,7 MPa;

- La resistencia media a tracción de los bloques de adobe estudiados se sitúa en torno al $20 \%$ de la respectiva resistencia a compresión;

- El análisis granulométrico de los áridos de los bloques de adobe recogidos en la región puso de manifiesto la naturaleza arenosa de las tierras constituyentes de los mismos, sin aptitud para usarse aisladamente como material de construcción, resultando por ello imprescindible la estabilización de los mismos para su uso en la fábrica de adobe, la cual, tradicionalmente se realizó con adición de cal.

Fueron realizados ensayos en muros de fábrica de adobe cuyo objetivo era el de caracterizar y facilitar el análisis de soluciones de mejora del comportamiento de las estructuras de fábrica de adobe, ante las acciones horizontales. Los resultados alcanzados se constituyen como una herramienta de apo- 
yo a la identificación e interpretación de los daños estructurales más comunes, así como un instrumento de calibración de modelos numéricos que permite la evaluación de la seguridad y el desarrollo de soluciones de refuerzo. Además, cabe señalar que, este trabajo, si se extiende a otros casos de estudio podrá permitir caracterizar los modos de rotura de paredes fuera del plano, el nivel de deformación admisible y caracterizar el comportamiento histéretico de este tipo de fábrica.

De los ensayos realizados, resulta igualmente importante el comportamiento frágil que se ha observado en las fábricas de adobe estudiadas con solicitación horizontal. Hecho que constituye un problema para los edificios existentes, debido a su localización en zonas de moderada a alta peligrosidad sísmica. Con el trabajo de laboratorio realizado, se obtuvieron resultados que contribuyen a la evaluación del funcionamiento de las estructuras de fábrica de adobe cuando sometidas a cargas cíclicas, como las provocadas por los sismos. Así pues, señálese que con el trabajo experimental realizado se obtuvieron conocimientos que pueden contribuir a la evaluación del funcionamiento estructural de las fábricas de adobe, al ser sometidas a cargas cíclicas, como las provocadas por los sismos. En efecto, con la obtención de la evolución tensión-drift del panel de la pared doble-T en su estado original fue posible caracterizar las deformaciones máximas admisibles, los modos de rotura y la capacidad resistente en corte de las paredes de adobe y se pudo evaluar aun la eficacia de una solución de reparación y refuerzo sísmico. Los ensayos realizados en la pared reforzada, permitieron igualmente concluir que algunos de los daños que suelen encontrarse en estas fábricas, se pueden reparar eficientemente con técnicas simples y de bajo coste. Otros trabajos desarrollados en la PUCP, Lima, Perú (11), han verificado también la eficiencia de este tipo de soluciones de refuerzo. Además, con estas soluciones es posible obtener una mejor respuesta y desempeño sísmico de este tipo de fábrica. Lo corroboran los resultados del ensayo de la pared rehabilitada, donde se confirmó la recuperación de la rigidez inicial, un aumento de la ductilidad de hasta el doble, una mejora bastante significativa en la capacidad de disipación de energía, un incremento de alrededor del $20 \%$ de la capacidad resistente al corte de la pared, y la eliminación del modo de rotura frágil, típica en este tipo de fábrica.

Finalmente, se hace notar que, la realización del trabajo descrito en este artículo está también destinada a inculcar en la sociedad una mayor conciencia para la problemática de la conservación de estas estructuras, tratando de, por la ampliación del conocimiento sobre el tema, permitir el acceso a información técnica, por parte de un conjunto variado de agentes involucrados en la preservación de este patrimonio, que permita la puesta en marcha a larga escala de la rehabilitación de las construcciones existentes.

En relación con el trabajo en curso, cabe señalar que está todavía por concluir el trabajo exhaustivo de caracterización que se expone en el presente artículo. El enfoque global planteado se basa en tres niveles (10). Se han establecido inicialmente contactos con todos los alcaldes de la región con objeto de recabar información sobre las zonas donde la construcción de adobe es más abundante (nivel 1). Basándonos en esta información básica fueron seleccionadas un gran número de zonas representativas de la realidad constructiva en adobe de la región, con objeto de profundizar aún más el estudio hasta aquí realizado (nivel 2) (Figura 49-a). La información y los conocimientos resultantes de estas fases de investigación (nivel 1 y 2) permitirán la elección fundamentada de los edificios más representativos que posteriormente serán objeto de una caracterización más detallada (nivel 3) a nivel patológico y de los sistemas constructivos. Esta caracterización se aplicará mediante fichas de inspección y evaluación adecuadamente elaboradas para este fin y ya validadas (Figura 49-b).
Agradecimientos

Los autores de este trabajo agradecen a todas las personas, empresas e instituciones que vienen colaborando en la preparación y realización de los ensayos, ayuda fundamental para los resultados obtenidos, en particular al Laboratorio de Ingeniería Sísmica y Estructural (LESE), de la Facultad de Ingeniería de la Universidad de Oporto, a la empresa STAP, y al ayuntamiento de la ciudad de Aveiro.

\section{BIBLIOGRAFÍA}

(1) Varum, H.; Costa, A.; Velosa, A.; Martins, T.; Pereira, H.; Almeida, J.: "Caracterização mecânica e patológica das construções em adobe no distrito de Aveiro como suporte em intervenções de reabilitação". Projecto Culture 2000/Mediterrae - Houses and cities built with earth: conservation, significance and urban quality (2006), pp. 41-45, ISBN 972-8479-41-7, Argumentum.

(2) Martins, T.; Varum, H.: "Adobe's Mechanical Characterization in Ancient Constructions: The Case of Aveiro's Region". Materials Science Forum, Vols. 514-516 (2006), pp. 1571-1575. Trans Tech Publications, Switzerland, ISSN 0255-5476.

(3) RILEM: "Technical Recommendations for the Testing and Use of Construction Materials". E\&FN Spon, UK, 1994. 
(4) Varum, H.; Costa, A; Pereira, H.; Almeida, J.: "Comportamento estrutural de elementos resistentes em alvenaria de adobe". TerraBrasil2006, I Seminário Arquitetura e Construção com Terra no Brasil y IV Seminário Arquitectura de Terra em Portugal (2006). Ouro Preto, Minas Gerais.

(5) Pereira, H.: Caracterização do comportamento estrutural de construções em adobe. Tesis de Maestría en Ingeniería Civil. Departamento de Engenharia Civil, Universidade de Aveiro, Aveiro, 2008.

(6) Figueiredo, A.: Caracterização de uma solução de reforço sísmico de paredes de adobe. Tesis de Maestría en Ingeniería Civil. Departamento de Engenharia Civil, Universidade de Aveiro, Aveiro, 2009.

(7) Tareco, H.; Grangeia, C.; Varum, H.; Senos-Matias, M.: "A high resolution GPR experiment to characterize the internal structure of a damaged adobe wall". EAGE First Break, Vol. 27, N. 8 (2009), pp. 79-84.

(8) Costa, A.; Varum, H.; Pereira, H.; Rodrigues, H.; Vicente, R.; Arêde, A.; Costa, A.A.: "Avaliação experimental do comportamento fora do plano de paredes de alvenaria de adobe". V Seminário Arquitectura de Terra em Portugal, (2007) Departamento de Engenharia Civil, Universidade de Aveiro, Aveiro. ISBN 978-989-20-0903-2.

(9) Varum, H.; Costa, A.; Pereira, H.; Almeida, J.; Rodrigues, H.: "Caracterização do comportamento estrutural de paredes de alvenaria de adobe". Mecânica Experimental, Vol. 15 (2008) pp. 23-32. APAET, Associação Portuguesa de Análise Experimental de Tensões. ISSN 1646-7078.

(10) Silveira, D.; Varum, H.; Costa, A.: "Rehabilitation of an important cultural and architectural heritage: the traditional adobe constructions in Aveiro district". Sustainable Development (2007), pp. 705-714. Editors: A. Kungolas, C.A. Brebbia, E. Beriatos - WITPress, Carvoeiro, Algarve. ISBN 978-1-84564-103-0.

(11) Blondet, M.; Torrealva, D.; García, G.; Ginocchio, F.; Madueño, I.: Using Industrial Materials for the Construction of Safe Adobe Houses in Seismic Areas. PUCP, Peru, 2004. 